\title{
Orthodontia-implantology- prosthodontics in rare diseases: the oligodontia example
}

\author{
Khanh NHAM, Steve TOUPENAY, Dac Alexandre VUONG, \\ Nadeem SAMEE, Benjamin FOURNIER, Stéphane KERNER
}

\begin{abstract}
Among rare diseases, a fifth of them are associated with oral manifestations. The most frequent are: enamel dysplasia, cleft lip or palate and oligodontia. Beside the pathology, patients are eager of functional therapeutics which often needs prosthodontics. This oral rehabilitation aims to a better life quality.

Teeth absence management in the case of oligodontia necessitates critical analysis of the supporting tissues: the periodontium and the underlying bone. Deciduous teeth with resorbed roots and hypoplasic permanent teeth are often observed. Thus, some questions arise: can we use them as abutments or can we restore them knowing that they present an anomaly in their position and are more fragile? How do we replace many teeth when bone is lacking (clefts, agenesis)? Can we implant on a missing tooth site or can we use autograft? What do we expect for periodontal healing?

Adjacent teeth have often migrated, occlusion is unstable due to mixed dentition and patient's disease. For example, in mandibular incisors agenesis cases, the remaining deciduous teeth are used when consultation happens at adolescence. This results in upper jaw incisors migration toward mandible and thus an anterior overbite. Orthodontic treatments will allow to recreate gap width and a normal occlusion in order to perform prosthodontics treatment in the best possible conditions. Giving the weak dental and periodontal supports, our attention will be focused on choosing the best option between tooth and implant supported prosthodontics. During the mixed dentition, orthodontic treatment and aesthetic rehabilitation have to be planed at the same time and to last until "definitive" implant and periodontal therapies are possible. In this article, we will present one oligodontia case report to illustrate a possible solution.
\end{abstract}

KEY WORDS

Orthodontics implantology prosthesis oligodontia 


\section{INTRODUCTION AND DESCRIPTION OF THE REFERENCE CENTER}

Since its designation in 2007 as a Reference Center of Rare Disease for the Face and Oral Cavity (MAFACE), Rothschild Hospital has treated 631 patients including $57 \%$ with congenitally missing teeth ${ }^{23}$. The annual number of new patients is approximately 100 , about half of them present with an abnormality in the number of teeth (internal data from the MAFACE reference center 2012). The management of these patients at the MAFACE reference center is complex and requires multidisciplinary treatment.

This article addresses the close cooperation that exists between orthodontics and implant-prosthetics and focuses on the therapeutic management of patients presenting with agenesis of multiple teeth, particularly, oligodontia. This disorder is characterized by agenesis of 6 or more permanent teeth, excluding wisdom teeth ${ }^{14}$. According to different studies, the prevalence of agenesis of permanent teeth can vary from $.09 \%$ to $.14 \%{ }^{2,18}$. Several rare disorders, whether syndromic or not, include agenesis of multiple teeth in their clinical picture, with ectodermal dysplasia as the most commonly mentioned $^{11}$. The early diagnosis and therapeutic management of these rare disorders requires prosthetic and orthodontic follow-up care in order to ensure an ideal clinical situation so that when the patient becomes an adult, it will then be possible to provide a reliable restorative treatment. The common objective of any treatment is functional and esthetic restoration. But the specificity of these pathologies comes from the need to time the treatment to the stages of growth, child, adolescent and then young adult using the appropriate technical as well as psychological approach. Accommodating patients transitioning into adulthood in the best way possible during prosthetic restoration and implant treatment will help them to accept the treatment and help the practitioner to have the best conditions to perform it so that it lasts throughout the lifetime.

Coordination within the MAFACE reference center requires the intervention of specialists in dento-facial orthopedics, general dentistry and maxillofacial surgeons who adapt treatment to the clinical picture and psychological make-up of these patients. Conventional advances in specific therapeutic management of agenesis of multiple teeth have made it possible to establish a specific protocol for treatment for children since 2007, and for adults since 2012. Therefore, the nomenclature confers legal recognition to patients with oligodontia who then qualify for reimbursement of costs for implant treatment after the genetic defect has been confirmed by an expert at the center for expertise, as requested by the division of rare diseases of the National Health Insurance Fund. The available scientific literature is limited to the opinions of experts or to case series. This represents limited scientific proof ${ }^{17}$. 


\section{CHARACTERISTICS OF OLIGODONTIA}

\section{Dentoalveolar characteristics}

A review of the literature shows some common dento-skeletal characteristics in these patients.

- The developmental defect of the dental organ may be linked to a lack of maturation or lack of tooth buds (defect in genetic coding or embryopathology).

- The most frequently involved teeth are those at the end of a tooth series ${ }^{9}$.

- When they are present, deciduous teeth erupt unpredictably. They may either persist in a stable manner in the arch with no radicular breakdown or on the contrary fall out or root resorption may even begin and eventually there will be ankylosed teeth in the $\operatorname{arch}^{12}$, and this takes place, despite the absence of succedaneous tooth buds. Primary molars may then become submerged, and consequently, tooth elongation of the opposing dentition occurs ${ }^{16}$.

- Structural abnormalities may be detected such as dysplasia that can vary in severity.

- The co-existence in the arch of such hypotrophic primary and permanent teeth is possible, creating interdental gaps that are difficult to manage. We can observe variations in the mesiodistal dimensions even though they may not appear significant as well as a morphological leftright asymmetry of teeth in patients with oligodontia ${ }^{20}$. These variations may be found in the relatives of these patients. This abnormality of shape might be more marked in the case of oligodontia that shows a noticeable difference in tooth dimension for the maxillary lateral incisors and the mandibular canines ${ }^{10,24}$.

- In the labial-lingual direction, the dimensions of the alveolar process are reduced in the area of agenesis, whether or not primary teeth are present. The presence of crowns and dental roots is important for the stimulation of the development and the quality of the alveolar process, since their absence can complicate implant treatment procedures. This is even more flagrant when there is an early loss of deciduous teeth that leads to a bone defect.

\section{Dentofacial characteristics of oligodontia}

- On the sagittal plane, several studies $^{1,25}$ suggest that a retruded chin and a very open interincisal angle are present. This explains the lingual inclination of the mandibular incisors that is often combined with palatal inclination of maxillary incisors. The profile is therefore very straight or even concave with prognathia.

- The absence of teeth, especially in the lateral sectors, often includes a decrease in the height of lower third of the face with significantly more prominent 
nasal and chin furrows as well as an incisal overjet. It is not unusual to see even more prominent wear facets appear on the incisors if primary teeth affected by agenesis are involved.

The research of Marijn Créton et al. ${ }^{8,9}$ on cases of dental agenesis indicates that, although bone trabeculation shows no variations in either healthy or oligodontia patients, there are three interesting discrimatory cephalometric variables: the sagittal relationships of the maxilla (SNA, SNB), the intercisal angle and the height of the lower third of the face (possibly the relationships of the heights of each third as described by the Wendell-Willie Equation). Patients can be grouped into different categories:

- loss of few teeth with little impact on the face;

- loss of 4.3 teeth on average with labial inclination of the mandibular incisors;

\section{ORTHODONTIC TREATMENT}

Orthodontic treatment management in cases of oligodontia may be comprised of two phases: the management of edentulous spaces and a more comprehensive dentofacial restoration.

In the first case, the number of missing teeth is relatively reduced and involves the end of series teeth (classically second premolars and maxillary lateral incisors). The repercussions on the support tissue are not as great. The management of the space beyond a possible arch length discrepancy due to excess space
- lingual inclination of the incisor in both arches with loss of 6 teeth on average including the maxillary premolars;

- absence of 7 teeth or more including the molars with retruded chin.

- A reduction in the length of the maxillary and mandibular arches respectively of $4.40 \mathrm{~mm}$ and $2.80 \mathrm{~mm}$ may also be observed in patients presenting oligodontia. The intercanine distance is decreased by $2.82 \mathrm{~mm}$ in the maxilla and by $2.70 \mathrm{~mm}$ in the mandible, and the intermolar distance is also decreased by $3.40 \mathrm{~mm}$ in the maxilla and $1.80 \mathrm{~mm}$ in the mandible ${ }^{6 .}$

These clinical characteristics make orofacial restorations complicated and compromises are often necessary.

will take place in concert with a periodontal-prosthetic restoration with a choice between a bridge or an implant, closure or reopening of the spaces as in this case, may cause a divergence of the roots in the collateral teeth. The occlusal scheme may show a discrepancy in the normal sagittal relationships of the teeth if there are a limited number of teeth that will be replaced (for example: Class II therapy with agenesis of the lateral incisors). Of course, the practitioner must take into consideration potential abnormalities of shape or structure when 
deciding on treatment. The principal difficulty of orthodontic treatment for patients with oligodontia will be the anchorage system and the quality of the supporting bone. The permanent and primary teeth present in the mouth are often distant from one another that subsequently creates diastemas. In addition, they may present with reduced coronal volume. It is often necessary to divide the mouth into different zones for procedures in order to create more solid supports and anchorage devices such as miniscrews are an indispensable tool. However, in the previously edentulous areas, the weakness of the bone support, both in the buccal-lingual direction as well as in the coronal-apical direction, requires that the orthodontist takes every precaution during dental movements by using gentle and continuous force to avoid the risk of exposing the roots. This is especially true when congenitally missing teeth occur as part of a syndrome such as cleft of lip and palate with regard to the closure of surgical margins. Sometimes, the decision is made to preserve the primary teeth, either to prevent loss of the support until the patient is old enough to insert the implants or to mask them with composite or resin veneers. In this case, during orthodontic movement every precaution should be taken given that root resorption may occur.

\section{IMPLANT PROSTHETIC TREATMENT}

As in any complicated situation and based on the principle that implants guide surgery, making a diagnostic wax up on study models and a radiographic guide are indispensable
It is often necessary to perform coronal reconstruction beforehand, with either pediatric crowns for the molars or micro-filled composite veneers for the anterior zone. The reconstruction contributes substantially to the reliability of the placement of orthodontic brackets and to their durability throughout treatment that allows for better control.

In cases of more extensive missing teeth, the facial repercussions on the support tissues often initially require the fabrication of occlusal splints in order to confirm the rehabilitation of the height of the lower third of the face and as well as the restoration of the support for the desired labial and cheek-side structures. The participation of the patient in the process of indicating satisfaction with the sequential outcomes is essential to the acceptance of achieved results. A profile cephalometric Xray will help to confirm the correct inclination of the condylar heads and will be reconfirmed by a new height that is symptom free.

Of course, this is just an example of treatment management for isolated oligodontia, the treatment plan becomes more complicated in cases of associated sagittal imbalance and the practitioner and patient may opt for a traditional orthognathic surgery treatment plan.

along with a CT-scan in order to visualize bone volume and to plan for a bone graft if needed. The bone graft may be accomplished with onlay autogenous grafting or with a bone 
substitute material. They may come from mandibular, coxal or parietal bones.

In the buccal-lingual and mesialdistal direction, choosing narrowdiameter implants is an alternative ${ }^{7}$. This requires that the dental abutment be customized in relation to the desired emergence profile of the teeth. If an insufficient alveolar ridge in the mesial-distal plane can be managed by orthodontic opening of the space, the buccal-lingual plane need only be treated surgically by increasing the bone volume.

\section{IMPACT OF GROWTH ON REHABILITATION}

The prosthetic treatment represents the final phase of oral rehabilitation. There is a whole therapeutic arsenal available for restoring the function and esthetics of the patient centered around either fixed prosthetic restorative solutions or with removable solutions with or without the use of retention devices.

With regard to prosthetics, the practitioner will have to take into account two fundamental criteria: the age of the patient as well as his dental and skeletal structure, as determined by the intrinsic and extrinsic value of each tooth.

These patients, once they learn about the existence of new legal provisions concerning treatment for agenesis of multiple teeth, come in at any age for consultation. Since real age is not the main criterion to consider but rather the dental and skeletal maturation according to Thilander ${ }^{22}$, there are
In the vertical direction, the lack of dental development can lead to a significant deficiency of the height of the alveolar bone and therefore it becomes impossible to have the standard 8 to $10 \mathrm{~mm}$ total length used for an implant, with a higher risk of damaging structures such as the lower alveolar vascular nerve plexus or the maxillary sinus membrane. In this case, inserting short implants (less than $8 \mathrm{~mm}$ ) may be an alternative to vertical bone grafting and the placement of standard sized implants ${ }^{19}$. then three different age categories for prosthetic rehabilitation: adolescents, young adults and adults. Although treatment plans are individualized, it is best to treat patients as soon as possible.

Treatment for adolescents consists in preparing the dental arch for a prosthetic rehabilitation at the end of the growth period. The treating practitioner, in collaboration with the orthodontist, plans for the replacement of missing teeth. Based on the prosthetic procedure, the dentist decides to maintain, to close or to open the space. In cases of a single missing tooth in one quadrant in adolescents, an adhesive technique with a bonded prosthesis is preferable. On the one hand, this make it possible to avoid removable appliances and their disadvantages, and on the other to maintain the interdental space, whether or not it was created by 
the orthodontist, for implant treatment when growth has definitively stopped.

In severe cases of oligodontia, or anodontia, inserting implants before the end of skeletal development is not recommended, except in the symphyseal region. It is only advisable to place from two to four symphyseal implants in children ages 6 to 18 in cases where the practitioner is planning a rehabilitation with an implant-supported removable complete dentures, if wearing a single implant overdenture has proved unsuccessful ${ }^{11,13}$.

The comprehensive strategy for treating a young adult with oligodontia, is to suggest replacement therapy, while at the same time respecting the craniofacial development of the patient. Bio-adaptable partial implants, guidance for the eruption of permanent teeth, and closing or opening spaces between the permanent teeth for a permanent fixed solution at the end of the growth period, prevents any iatrogenic damage to the residual teeth. If spacing has to be maintained, it will generally be done by removable and bio-adaptable prostheses that should be monitored and reassessed at regular intervals, based

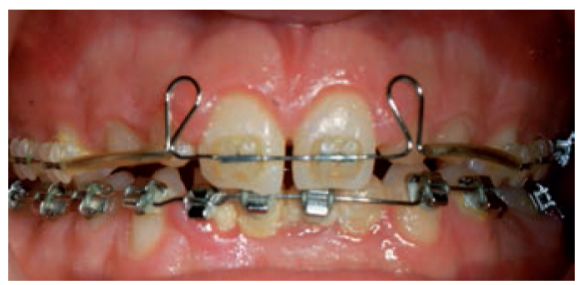

Figure 1

Clinical view during the course of DFO treatment (DFO treatment performed by Dr. Reveret). on the eruption of the permanent teeth and on the mobility of the adjacent teeth during orthodontic treatment. The other short-term solutions may be fixed using temporary devices attached to adjacent teeth by bonded wires, or temporary teeth integrated in the orthodontic arc. The case of Mrs. M. (Figs. 1 to 11) illustrates perfectly how orthodontics, implantology and prosthetics had to be coordinated in this case of agenesis where the decision was made to conserve the maximum number of primary teeth in the mandible and to reopen the spaces of the lateral incisors and canines in preparation for an implant-supported replacement.

Implant-prosthesis rehabilitation for young adults is possible at the end of puberty, but according to a study carried out by Bernard ${ }^{4}$, the coronal reconstruction is at risk of developing an infraclusion in the long term. Therapy initiated too early can also have an impact on the development of the arches, and on the eruption and position of adjacent toothbuds ${ }^{15}$. The risk of periodontal recession on the cervical edge of an anterior prosthesis in a young adult forces us to position the implants more distally and to opt for masking the natural teeth with esthetic cosmetics and a coronoplasty

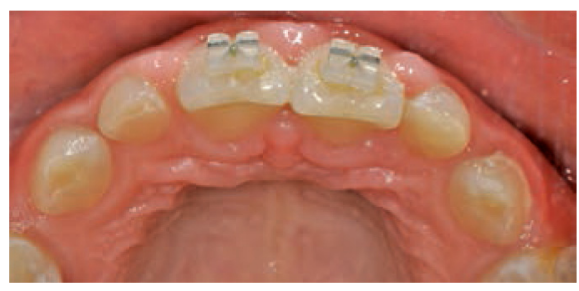

Figure 2

Occlusal view. 


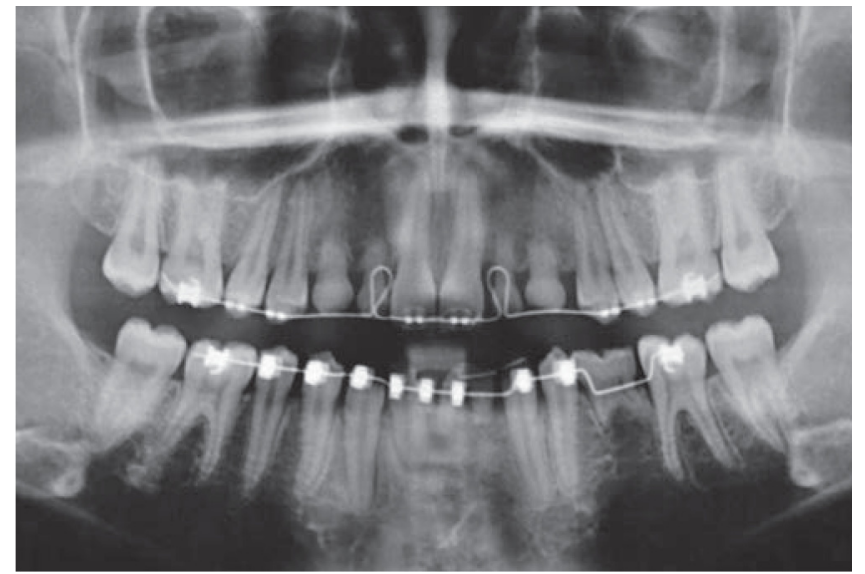

Figure 3

Panoramic xray at the end of orthodontic treatment.

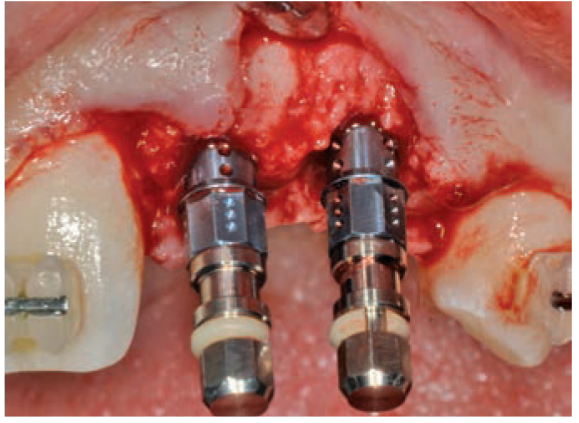

Figure 4

Insertion of implants in tooth positions 2223.

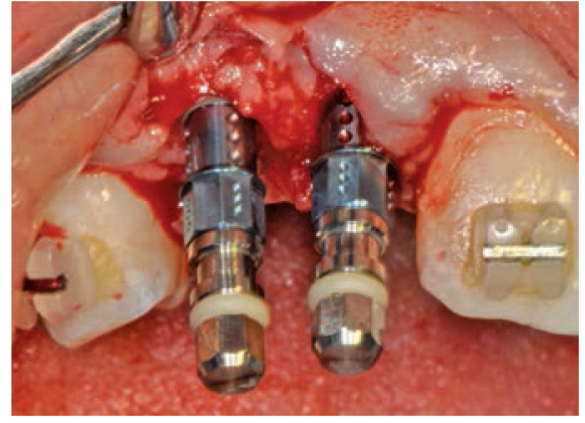

Figure 5

Insertion of implants in tooth positions 1213.

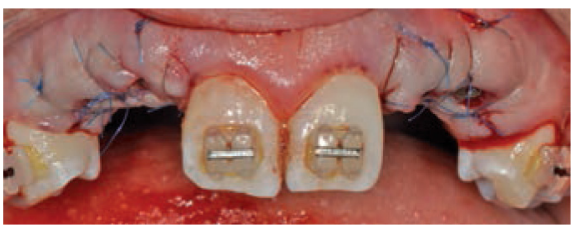

Figure 6

Sutured flaps with positioning of buried connective tissue grafts in a frontal view.

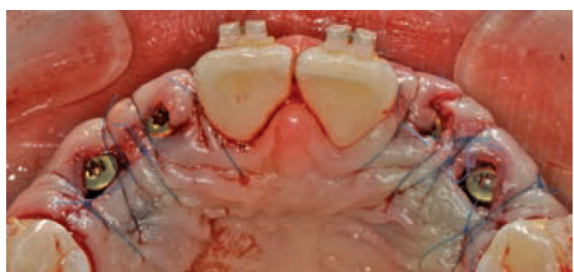

Figure 7

Sutured flaps with positioning of buried connective tissue grafts in a palatal view.

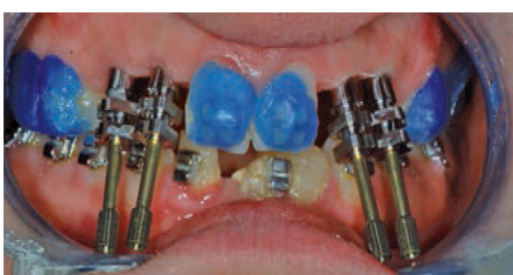

Figure 8

Placement of impression copings and protecting the brackets with wax. 


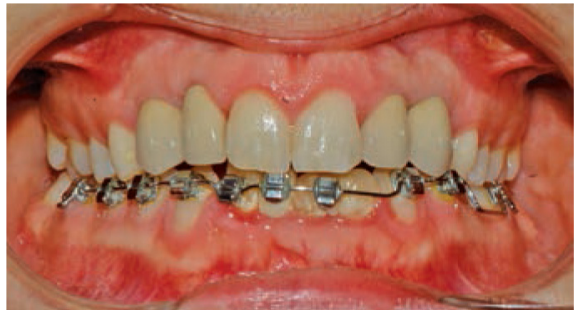

Figure 9

Prosthesis used in a frontal view.

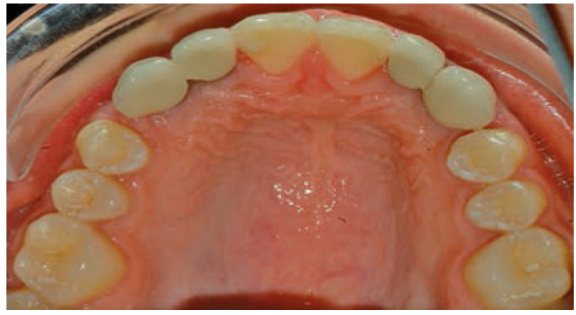

Figure 10

Prosthesis used in a palatal view.

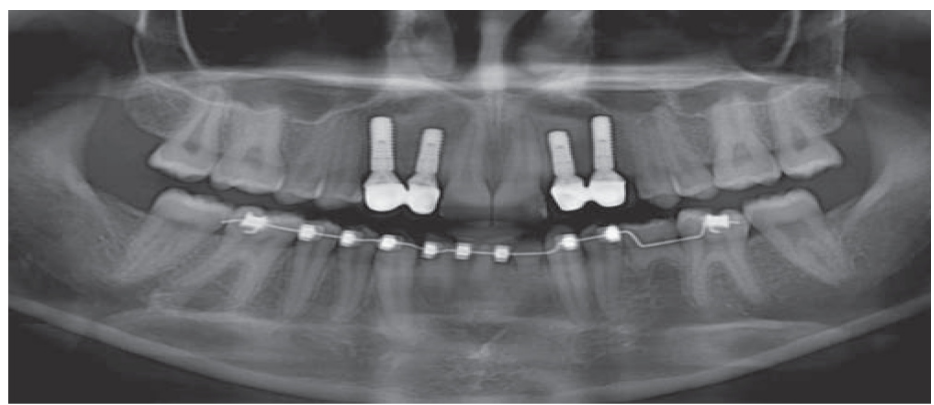

Figure 11

Panoramic xray of the teeth at the end of comprehensive treatment.

to ensure better matching with the replaced tooth.

In the adult patient, depending on the diagnosis, his complaints and his motivation, the potential solutions may involve an orthodontic procedure prior to the oral rehabilitation. If there is no preprosthetic orthodontic treatment, the practitioner must strive to be as uninvasive as possible and to adapt treatment to the existing occlusion, by looking for the best esthetic and functional compromise for the patient. In addition to the conventional therapeutic arsenal, patients with oligodontia can take advantage of special financing for implant therapy as well as a screw retained overdenture prosthesis that is a less expensive rehabilitation for a patient with oligodontia.
In cases where an orthodontic treatment is planned, treatment management is more comprehensive and long. The majority of patients with oligodontia present with a combination of primary and permanent teeth in the arches, and the question of conserving the primary teeth in preparation for a prosthetic rehabilitation must be considered. The extraction of primary teeth is not systematically performed. If the primary second molars are present at 20 years of age, their prognosis for survival in the long term is improved ${ }^{5}$. Studies show then the possibility of maintaining the primary molars in adults, especially primary second molars that subsequently makes it possible to delay implant therapy ${ }^{21}$. If, in spite of everything, they must be extracted 
and implant-supported solution is elected, the orthodontic and prosthetic plan will be a preparation for the implant insertion ${ }^{3}$. Since adult prosthetic solutions are identical to those for young adults, an orthodontic treatment, as decribed above, will promote the integration of the suggested prosthetic elements.

\section{CONCLUSION}

The various forms of oligodontia included in the category of agenesis of multiple teeth consist of several clinical manifestations. Depending on the number of missing teeth, their distribution in the arches, the occlusal picture may be disrupted to varying degrees (extrusion, linguoversion) and the affected support tissue (receded chin, reduction of the perimeter of the arch, collapsed labial and cheek tissues).

Clinical therapeutic management must necessarily be comprehensive and planned in several phases over time based on the age of the patient. The management must also have a psychological component so that the patient, who has so many esthetic and functional needs, can easily anticipate the various phases and accept the need to cooperate as well as the outcome of treatment. Reviewing the oral rehabilitation plan on mock-ups may be a means of winning the support and cooperation of the patient, and in the same way may help the medical team clearly understand the desired results. The need for cooperation between dentofacial orthopedics and prosthodontics is of the utmost importance to ensure the reliability of the rehabilitation, performed according to the latest scientific data available, in order to therefore avoid the inherent medical-legal implications of "lost opportunity" for these young patients.

\section{BIBLIOGRAPHY}

1. Ben-Bassat Y, Brin I. Skeletal and dental patterns in patients with severe congenital absence of teeth. Am J Orthod Dentofac Orthop Off Publ Am Assoc Orthod Its Const Soc Am Board Orthod 2009;135(3):349-56.

2. Bergendal $B$, Norderyd J, Bågesund $M$, Holst A. Signs and symptoms from ectodermal organs in young Swedish individuals with oligodontia. Int $\mathrm{J}$ Paediatr Dent $\mathrm{Br}$ Paedodontic Soc Int Assoc Dent Child. 2006;16(5):320-6.

3. Bergendal B. When should we extract deciduous teeth and place implants in young individuals with tooth agenesis? J Oral Rehabil 2008;35:55-63.

4. Bernard JP, Schatz JP, Christou P, Belser U, Kiliaridis S. Long-term vertical changes of the anterior maxillary teeth adjacent to single implants in young and mature adults. A retrospective study. J Clin Periodontol 2004;31(11):1024-8.

5. Bjerklin $\mathrm{K}$, Bennett $\mathrm{J}$. The long-term survival of lower second primary molars in subjects with agenesis of the premolars. Eur J Orthod 2000;22(3):245-55.

6. Bu X, Khalaf K, Hobson RS. Dental arch dimensions in oligodontia patients. Am J Orthod Dentofac Orthop Off Publ Am Assoc Orthod Its Const Soc Am Board Orthod. 2008;134(6):768-72. 
7. Chiapasco M, Casentini $P$, Zaniboni M, Corsi $E$, Anello T. Titanium-zirconium alloy narrow-diameter implants (Straumann Roxolid $\left({ }^{\circledR}\right)$ for the rehabilitation of horizontally deficient edentulous ridges: prospective study on 18 consecutive patients. Clin Oral Implants Res 2012;23(10):1136-41.

8. Créton M, Geraets W, Verhoeven JW, van der Stelt PF, Verhey H, Cune M. Radiographic features of mandibular trabecular bone structure in hypodontia. Clin Implant Dent Relat Res 2012;14(2):241-9.

9. Créton M, Cune MS, de Putter C, Ruijter JM, Kuijpers-Jagtman AM. Dentofacial characteristics of patients with hypodontia. Clin Oral Investig 2010;14(4):467-77.

10. Gungor AY, Turkkahraman H. Tooth sizes in nonsyndromic hypodontia patients. Angle Orthod 2013;83(1):16-21.

11. HAS Service évaluation des actes professionnels. Traitement des agénésies dentaires multiples liées aux dysplasies ectodermiques ou à d'autres maladies rares, chez l'enfant atteint d'oligodontie. HAS, Service évaluation des actes professionnels ; 2006 décembre.

12. Haselden K, Hobkirk JA, Goodman JR, Jones SP, Hemmings KW. Root resorption in retained deciduous canine and molar teeth without permanent successors in patients with severe hypodontia. Int J Paediatr Dent Br Paedodontic Soc Int Assoc Dent Child 2001;11(3):171-8.

13. Heuberer S, Dvorak G, Zauza K, Watzek G. The use of onplants and implants in children with severe oligodontia: a retrospective evaluation. Clin Oral Implants Res. 2012;23(7):827-31.

14. Hobkirk JA, Brook $\mathrm{AH}$. The management of patients with severe hypodontia. J Oral Rehabil 1980;7(4):289-98.

15. Kramer F-J, Baethge C, Tschernitschek H. Implants in children with ectodermal dysplasia: a case report and literature review. Clin Oral Implants Res 2007;18(1):140-6.

16. Kurol J, Thilander B. Infraocclusion of primary molars with aplasia of the permanent successor. A longitudinal study. Angle Orthod 1984;54(4):283-94.

17. CEBM Centre For Evidence Based Medicine. Oxford Centre for Evidence-based Medicine - Levels of Evidence (March 2009) [Internet]. CEBM Cent Evid Based Med. Available from: http://www.cebm.net/?o=1025

18. Polder BJ, Van't Hof MA, Van der Linden FPGM, Kuijpers-Jagtman AM. A metaanalysis of the prevalence of dental agenesis of permanent teeth. Community Dent Oral Epidemiol. 2004;32(3):217-26.

19. Renouard F, Nisand D. Impact of implant length and diameter on survival rates. Clin Oral Implants Res 2006;17 Suppl 2:35-51.

20. Schalk-van der Weide Y, Steen WH, Beemer FA, Bosman F. Reductions in size and left-right asymmetry of teeth in human oligodontia. Arch Oral Biol 1994;39(11):935-9.

21. Sletten DW, Smith BM, Southard KA, Casko JS, Southard TE. Retained deciduous mandibular molars in adults: a radiographic study of long-term changes. Am J Orthod Dentofac Orthop Off Publ Am Assoc Orthod Its Const Soc Am Board Orthod 2003;124(6):625-30.

22. Thilander B, Odman J, Gröndahl K, Friberg B. Osseointegrated implants in adolescents. An alternative in replacing missing teeth? Eur J Orthod 1994;16(2):84-95.

23. Toupenay S. La prise en charge des maladies rares en odontologie : un enjeu de santé publique. Paris 7 Garancière ; 2010.

24. Vasselet P. Analyse tridimensionnelle de I'anatomie dentaire coronaire : étude des patients atteints d'oligodontie. Paris 7 Garancière ; 2011.

25. Yüksel S, Uçem T. The effect of tooth agenesis on dentofacial structures. Eur J Orthod 1997;19(1):71-8. 